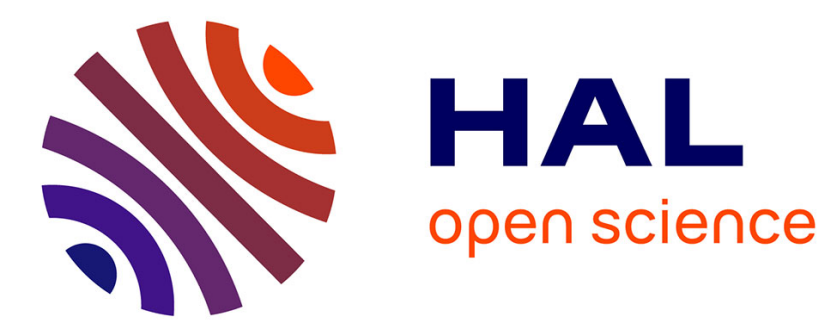

\title{
Le chiffre dans le gouvernement de la justice
}

Antoine Vauchez

\section{To cite this version:}

Antoine Vauchez. Le chiffre dans le gouvernement de la justice. Revue française d'administration publique, 2010, 10.3917/rfap.125.0111 . hal-02737006

\section{HAL Id: hal-02737006 \\ https://hal.science/hal-02737006}

Submitted on 23 Dec 2020

HAL is a multi-disciplinary open access archive for the deposit and dissemination of scientific research documents, whether they are published or not. The documents may come from teaching and research institutions in France or abroad, or from public or private research centers.
L'archive ouverte pluridisciplinaire HAL, est destinée au dépôt et à la diffusion de documents scientifiques de niveau recherche, publiés ou non, émanant des établissements d'enseignement et de recherche français ou étrangers, des laboratoires publics ou privés. 


\title{
LE CHIFFRE DANS LE « GOUVERNEMENT » DE LA JUSTICE
}

\author{
Antoine Vauchez
}

E.N.A. | Revue française d'administration publique

$2008 / 1-n^{\circ} 125$

pages 111 à 120

\section{ISSN 0152-7401}

Article disponible en ligne à l'adresse:

http://www.cairn.info/revue-francaise-d-administration-publique-2008-1-page-111.htm

Pour citer cet article :

Vauchez Antoine, «Le chiffre dans le « gouvernement » de la justice »,

Revue française d'administration publique, 2008/1 n 125, p. 111-120. DOI : 10.3917/rfap.125.0111

Distribution électronique Cairn.info pour E.N.A..

(c) E.N.A.. Tous droits réservés pour tous pays.

La reproduction ou représentation de cet article, notamment par photocopie, n'est autorisée que dans les limites des conditions générales d'utilisation du site ou, le cas échéant, des conditions générales de la licence souscrite par votre établissement. Toute autre reproduction ou représentation, en tout ou partie, sous quelque forme et de quelque manière que ce soit, est interdite sauf accord préalable et écrit de l'éditeur, en dehors des cas prévus par la législation en vigueur en France. II est précisé que son stockage dans une base de données est également interdit. 


\title{
LE CHIFFRE DANS LE « GOUVERNEMENT » DE LA JUSTICE
}

\author{
Antoine VAUCHEZ \\ Chercheur auprès du Centre Robert Schuman de l'Institut universitaire européen ${ }^{1}$
}

\section{Résumé}

Si le droit forme le langage commun et le terrain d'entente naturel des différents acteurs des politiques publiques de la justice, il partage désormais sa prééminence avec le chiffre qui fait figure de passage obligé des débats sur la réforme judiciaire. La conversion collective aux vertus du chiffre et des techniques quantitatives ne doit certainement pas laisser croire à une forme de pacification progressive des débats en la matière. Elle est d'abord l'occasion d'une recomposition des savoirs et des pouvoirs qui s'opère au détriment des formes d'autorégulation sociale qui caractérisait traditionnellement les mondes judiciaires.

\section{Abstract}

- Numbers in Judicial "Government" - While law constitutes the common language and natural common ground between the various actors in justice public policies, numbers are now considered equally important, and a vital aspect of the debates on judicial reform. The collective discussion on the virtues of numbers and quantitative techniques must clearly not give the impression that debates on the subject are gradually calming down. It is above all an opportunity for the reconstitution of knowledge and powers which is taking place to the detriment of the forms of social self-regulation that traditionally characterised judicial circles.

$33 \%$ des Français ; $2,13 \%$ du budget de l'État ; 4,70 euros par habitant ; 44e rang mondial ; 240 jours ; 77,9 \% ... Ces nombres, prix, pourcentages, moyennes, ratios, et classements, aussi différents soient-ils, ont un point commun. Ils appartiennent à la liste

1. antoine.vauchez@eui.eu 
désormais longue de ces chiffres phares qui forment le lot inévitable des diagnostics de la justice française. Ensemble, ils mesurent tour à tour l'indice de confiance des français, la part de la justice dans le budget de l'État, le montant de l'aide juridictionnelle par habitant, le classement de l'attractivité économique française, le nombre de jours pour traiter un vol avec violence, ou le taux de réponse pénale. Ces objectivations chiffrées de l'état de l'institution judiciaire occupent désormais presque invariablement les premiers rôles dans les discours académiques, politiques, bureaucratiques, juridiques sur la réforme. Ils entendent pointer tout à la fois la «crise de confiance des français dans l'institution judiciaire », son "manque de moyens », la nécessité de développer les systèmes d'assurance juridique privé, la «faible attractivité du système juridique français », la lenteur des procédures judiciaires, la judiciarisation voire "l'américanisation » de la société française, ou encore l'efficacité de la lutte contre l'insécurité. Longtemps objet de connaissances essentiellement impressionnistes et éparses, évoquées le plus souvent sur le mode lettré des "gens de justice », la justice s'est ainsi transformée au cours des quinze dernières années en enjeu de chiffre. Palmarès, sondages, cartes, logiciels comptables ou batteries d'indicateurs forment l'outillage minimal pour qui entend sérieusement envisager les paramètres de l'équation « réforme de la justice ». Traditionnellement considérés comme impropres à caractériser la fonction hautement symbolique de l'institution judiciaire, les termes de stock, flux, performance, rendement, coût ou efficacité en constituent aujourd'hui le champ lexical presque ordinaire. Difficile en somme d'imaginer aujourd'hui discours en la matière qui fasse l'économie du chiffre.

Certains d'entre eux ont acquis une valeur symbolique forte au cours des dernières années. Ainsi de la part de la justice dans le budget de l'État qui fait figure de point de repère de l'importance accordée par les gouvernements successifs aux questions judiciaires ; du taux d'augmentation du contentieux civil qui permet de suivre ce qui est perçu comme «l'explosion du contentieux »; ou encore, du "taux de réponse pénale » qui est devenu « l'indicateur phare » de la capacité du gouvernement à lutter contre l'insécurité 2 , etc. Élevé de la sorte au rang de levier privilégié pour la décision et le pilotage de la machine judiciaire, le chiffre apparaît fréquemment aussi comme un objectif en soi des politiques publiques de justice. La réforme en cours de la carte judiciaire constitue un terrain d'observation presque trop évident tant le souci rationalisateur y est ancien ${ }^{3}$. Désormais en effet, le chiffre n'est plus ni un outil ponctuel des réformes, ni un dispositif cantonné au seul domaine de l'organisation judiciaire. La loi organique relative aux lois de finances, on le sait, le place au cœur même du «pilotage » presque quotidien de la justice touchant désormais l'activité judiciaire elle-même par le biais des objectifs fixés en matière de réponse pénale, de rendement ou de «frais de justice ». Cette saillance du chiffre n'est d'ailleurs pas propre à l'administration. C'est en son nom qu'on se mobilise aujourd'hui dans les milieux juridiques. Le montant de l'unité de valeur de l'aide juridictionnelle est ainsi devenu depuis dix ans la principale pomme de discorde entre la Chancellerie (qui en fixe le niveau) et les organisations professionnelles des avocats. De même, il aura fallu le classement médiocre de la France par le rapport de la Banque mondiale sur l'attractivité économique des systèmes juridiques pour provoquer la mobilisation inédite des différents représentants de la « culture juridique française » dans une sorte de consortium promouvant, sous l'égide du Conseil d'État, les vertus des systèmes de civil law.

2. Voir par exemple, Guibert (Nathalie), «La réponse pénale, nouvel indicateur phare », Le Monde, 6 décembre 2006.

3. Commaille (Jacques), Territoires de justice. Une sociologie politique de la carte judiciaire, PUF, 2000. 
L'ambition de cet article n'est ni d'évaluer la qualité de ces chiffres, ni de faire le tri entre les usages qui en sont faits dans le débat public ${ }^{4}$. Il s'agit plutôt de saisir au travers de cette reformulation quantitative des termes du débat des transformations du " gouvernement » de la justice, c'est-à-dire des savoirs et des acteurs chargés à des titres divers d'en évaluer la légitimité et d'en préparer la réforme ${ }^{5}$. Et de fait, si cette conversion collective aux vertus du chiffre marque la montée en puissance du registre de l'objectivité et de la neutralité qui prime désormais sur la mise en avant des enjeux politiques de la réforme, elle ne doit certainement pas laisser croire à une forme de pacification progressive des débats. Relais essentiel d'une transformation du regard public sur la justice, le chiffre permet de suivre l'émergence de nouveaux experts et de nouvelles expertises en matière de justice en même temps que la remise en cause du modèle classique de régulation sociale des mondes judiciaires construit autour d'un idéal de singularité et d'incommensurabilité.

\section{LA MISE EN CHIFFRE DE LA JUSTICE}

La centralité du chiffre judiciaire est un phénomène récent indissociable en fait de l'essor d'un nouveau registre d'intervention publique dans les débats sur la justice - la «forme rapport»-mettant en avant objectivité et neutralité.

\section{Une conversion récente}

L'activité judiciaire a toujours généré du chiffre. On sait ainsi que la statistique criminelle a constitué l'un des premiers terrains sur lesquels s'est forgée l'appareil statistique de l'État français ${ }^{6}$. De même, la gestion des professions juridiques - qu'elle soit étatique pour les professions réglementées ou privée pour les professions libérales comme le barreau - s'est accompagnée du développement d'outils statistiques. Les divers organismes techniques de la profession d'avocat tels que la Caisse nationale des barreaux français (la Cnbf fondée en 1947) et l'Association nationale d'assistance administrative et fiscale des avocats (l'Anaafa créée en 1977) produisent ainsi de longue date des données quantitatives ${ }^{7}$. Mais cet équipement de la Chancellerie et des professions constituait pour l'essentiel un simple sous-produit des activités de gestion (recrutement, carrière, fonds de retraites... $)^{8}$. À ce titre, il restait pour l'essentiel non-public. Tandis que la

4. Ce qui ne signifie pas que ce travail n'est pas salutaire, sans doute tout particulièrement pour les raisons mêmes que souligne cet article. Voir en ce sens, Serverin (Évelyne), «De la statistique judiciaire civile et de ses usages », Revue internationale de droit économique, vol. 13, n² 2, 1999, pp. 281-294, et Aubusson (Bruno), Lalam (Nasser), Padieu (René), Zamora (Philippe), «Les statistiques de la délinquance », in Insee, France, portrait social 2002-2003, 2003, pp. 141-159. Voir aussi les publications de l'association Pénombre qui réunit depuis 1993 chercheurs, statisticiens et hauts fonctionnaires afin d'exercer une vigilance collective sur les usages du chiffre dans le débat public, notamment en matière pénale.

5. Cette mise en chiffre gagnerait à être mise en rapport avec le développement tous azimuts des standards qui, à l'image du développement de logiciels unifiés (de gestion des cabinets d'avocat, de gestion de l'aide juridictionnelle...), des certifications ISO des cabinets d'avocat ou de certaines juridictions, se diffusent depuis une quinzaine d'années dans l'univers judiciaire.

6. Desrosières (Alain), La politique des grands nombres. Histoire de la raison statistique, La découverte, 1993.

7. Vauchez (Antoine), Willemez (Laurent), Contribution à la connaissance statistique de la profession d'avocat, Rapport de recherche pour le Conseil national des barreaux, octobre 2002, $101 \mathrm{p}$.

8. Munoz Perez (Brigitte), «Les statistiques civiles, sous-produit du répertoire général des affaires civiles », Droit et société, $\mathrm{n}^{\circ} 25,1993$. 
statistique civile servait au mieux aux services du ministère pour leur permettre de suivre l'évolution de la carte judiciaire, la statistique criminelle était essentiellement utilisée pour saisir non pas tant les problèmes de la justice que l'état moral du pays ${ }^{9}$.

Le début des années 1990 marque de ce point de vue une véritable rupture. Tout se passe comme si le monde du droit et de ses professions s'était soudainement trouvé saisi par une forme de frénésie quantificatrice. La rareté de l'argument chiffré qui caractérisait un univers rétif à toute forme d'objectivation extra-juridique de son activité et de ses pratiques laisse place à une surabondance d'indicateurs et de données. Les données disponibles font désormais l'objet d'usages publics divers tandis que de nouvelles bases et de nouveaux instruments de mesure sont forgés qui alimentent une dynamique auto-entretenue de mise en chiffre de l'univers judiciaire. À défaut de pouvoir restituer ici plus en détail cette conversion progressive, on donne quelques repères chronologiques qui permettent de reconstituer une trame historique sommaire. Un premier moment de cet emballement a trait à la profession d'avocat elle-même. Avec l'instauration de l'aide juridictionnelle, l'État devient un contributeur essentiel du financement de la profession d'avocat et exerce de ce fait une influence considérable du point de vue salarial via la fixation annuelle par la Chancellerie du montant de l'unité de valeur de l'aide juridictionnelle. Dans ce cadre, la connaissance de la profession intéresse (au double sens du terme) les services du ministère de la justice. Dès 1994, la sous-direction des professions juridiques et judiciaires construit pour la première fois une base de données sur les avocats dont le périmètre se développe progressivement et fait l'objet depuis 1997 d'une publication annuelle disponible en ligne. Dans un contexte où les négociations avec le gouvernement sur l'aide juridictionnelle s'intensifient, les différents organismes représentatifs de la profession s'équipent à leur tour, à l'image du Conseil national des barreaux qui se dote en 2001 d'un Observatoire permanent de la profession chargé de constituer un annuaire statistique de la profession (notamment à partir des données statistiques disponibles via la Caisse nationale des barreaux français), suivi de près - comme il se doit - par le barreau de Paris 10 . Au-delà de ces protagonistes habituels des politiques publiques de la justice, un ensemble de nouveaux acteurs restés jusque-là à l'écart des débats sur la réforme de la justice y font désormais intrusion au nom du chiffre. Ce sont bien sûr les journalistes qui, palmarès et sondages d'opinion à l'appui, revendiquent désormais un point de vue surplombant sur les raisons et les enjeux de la crise de la justice. Ce sont les administrations centrales qui, dès 1999, par le biais des réflexions sur la qualité des services publics, puis de manière décisive après l'adoption de la loi organique relative aux lois de finances en août 2001, formalisent des «indicateurs de performance » construits sur la base des données statistiques disponibles ${ }^{11}$. Ce sont aussi les institutions internationales qui, converties pour des raisons diverses (développement économique, consolidation démocratique, efficacité administrative), à l'importance du bon fonctionnement de la machine judiciaire produisent un ensemble de classements pays par pays des performances. Chronologiquement, c'est la Banque mondiale ouvre le ban de manière spectaculaire. L'indice universel et synthétique de «facilité à faire des affaires » qu'elle crée en 2003 pointe désormais chaque année les

9. Chauvaud (Frédéric), « De la statistique morale à la statistique instrumentale. Jalons d'une réflexion critique sur les usages de la statistique judiciaire », Déviance et société, $\mathrm{n}^{\circ}$ 22, 1998, pp. 181-200.

10. Voir notamment «Un observatoire pour quoi faire ? ", in Observatoire du barreau de Paris, Statistiques 2001, 2002, p. 2. Voir aussi la publication annuelle des données de l'Anaafa dans la revue Maître.

11. La «mission justice » de la LOLF compte ainsi pas moins de vingt indicateurs de performance : inter alia, ancienneté moyenne du stock par type de juridiction, nombre d'affaires traitées par magistrat, délai moyen de traitement des procédures... 
effets négatifs de la lenteur, lourdeur, inefficacité et corruption pour la fluidité de la vie économique (contrats, conditions de liquidation des entreprises en difficulté...) ${ }^{12}$. Le palmarès qui en résulte et qui situe la France au 44e rang mondial est censé permettre d'identifier bons et mauvais «élèves » et de diffuser les « bonnes pratiques ». Bien que portée par une logique différente (la modernisation de l'État), la Commission européenne pour l'efficacité de la justice (CEPEJ) créée en 2002 au sein du Conseil de l'Europe, contribue à son tour à cette course au chiffre en produisant chaque année depuis 2004 un palmarès des «systèmes judiciaires européens » qui entend depuis s'imposer comme « un véritable outil au service des politiques publiques de la justice et des citoyens européens $» 13$.

\section{Le chiffre et son double : la « forme rapport»}

Ce déploiement du chiffre dans l'activité judiciaire ne se comprend pas sans évoquer l'émergence d'un nouveau registre de connaissance de la justice fondé sur la revendication d'une objectivité et d'une neutralité armées des outils de la science. Sans être absolument inédit, ce registre expert est longtemps resté marginal dans un univers où règnent traditionnellement en maître l'essayisme et le témoignage produits de l'intérieur de la machine par les professionnels de la justice eux-mêmes. Fondés sur l'expérience accumulée dans les prétoires, agrémentés de nombreuses anecdotes « édifiantes », ce type de diagnostics était souvent le fait de la plume éloquente et volontiers emphatique des «ténors du barreau». Ces « interventions » à chaud étaient sans doute plus rares dans le cas de la magistrature du fait de l'éthos de réserve et de secret qui fonde la définition classique de l'excellence professionnelle de ce corps ${ }^{14}$, mais il empruntait la forme détournée des mémoires, des histoires professionnelles quand ce n'était pas celle du libelle vengeur mais anonyme. L'avènement du fait syndical à partir de la fin des années 1960 aura contribué à diversifier les registres et les auteurs de cette littérature judiciaire ${ }^{15}$. Toute une production à visée explicitement politique émerge alors qui s'appuie sur des organisations (Syndicat de la magistrature, Syndicat des avocats français), des publics (militants) et des moments de mobilisation (congrès, manifestations...). On comprend aisément dès lors que le chiffre n'ait pas constitué l'argument de prédilection des débats sur la réforme.

Ce n'est en fait qu'avec l'émergence de la «forme rapport » 16 que l'argument du poids et des mesures fait son entrée dans les controverses. On a montré ailleurs combien s'étaient multipliés, à partir de la fin des années 1980, rapports, «livres blancs », et autres

12. L'indice synthétique de «facilité à faire des affaires » intègre dix indicateurs : création d'entreprises, obtention de licences, recrutement et licenciement, enregistrement de la propriété, obtention du crédit, protection des investisseurs, paiement des taxes, commerce transfrontalier, exécution des contrats, fermeture d'entreprises.

13. Commission européenne pour l'efficacité de la justice (CEPEJ), « Note explicative à la grille révisée pour l'évaluation des systèmes judiciaires », Strasbourg, 15 septembre 2005, site web de la Cepej (visité le 20 novembre 2007). La Cepej a constitué une grille d'évaluation comprenant 123 questions réparties en neuf chapitres : les données démographiques et économiques du pays, l'accès à la justice, l'organisation des tribunaux, les principes du procès équitable, les carrières des juges et des procureurs, les avocats, les modes alternatifs de règlement des litiges, l'exécution des décisions de justice, les notaires. Voir ici l'analyse du président du groupe des experts de la Cepej, Jean (Jean-Paul), « Approche méthodologique du rapport de la Cepej sur les systèmes judiciaires européens », consultable en ligne sur le site de la Mission Droit et Justice.

14. Sur cet ethos, voir Bancaud (Alain), «La réserve privée du juge », Droit et société, n 20-21, 1992, pp. 255-275.

15. Lenoir (Remi), « La parole aux juges », Actes de la recherche en sciences sociales, $\mathrm{n}^{\circ} 101-102$, mars 1994 , pp. 77-84.

16. Fournel (Jean-Louis), «Introduction à la "forme-rapport" : caractéristiques et temporalités d'une production de vérité publique », Cultures \& Conflits, n65, printemps 2007, pp. 37-49. 
états des lieux de la justice ${ }^{17}$. Si les institutions publiques - nationales et internationales - en sont les premiers commanditaires via les nombreuses commissions ministérielles, missions d'information, rapports d'inspection et commissions d'enquête parlementaires qu'ils ont formés au cours de la période, ils n'ont pas le monopole de cette forme d'intervention publique qui s'est progressivement diffusée aux fondations ${ }^{18}$, aux organisations non gouvernementales ( $c f$. Transparency international), mais aussi désormais - plus frappant encore - aux syndicats eux-mêmes ${ }^{19}$. Construit autour d'un travail d'enquête et parfois d'audition qui se veut méthodique, systématique voire scientifique, le rapport s'oppose trait pour trait à l'horizon étroit du témoignage individuel comme à l'appréhension jugée partiale du parti pris militant. À l'inverse de la subjectivité (le «je » du témoin) et de la politisation (le «nous » du collectif) propres aux formes de l'essai ou du pamphlet, il affiche une objectivité ( «la justice est... ») censée permettre d'établir un fait social «incontestable ». Dans la construction de cette «réalité » devant permettre de dépasser les intérêts «corporatifs » et les points de vue partisans, le chiffre fait figure de passage obligé. Dans le récit que tout rapport entend mettre en scène et qui lie étroitement le «diagnostic » des dysfonctionnements judiciaires aux propositions de réforme, les données quantitatives constituent invariablement le socle de départ. Ainsi du leitmotiv de «l'explosion du contentieux civil» (ou «explosion de la demande de justice »), véritable «ponts aux ânes » des rapports des années 1990 qui s'appuie sur la mobilisation, - souvent hâtive et imprécise -, de séries statistiques supposément édifiantes ${ }^{20}$. La « crise de la justice » telle qu'elle ressort de la forme - « rapport » apparaît ainsi comme le produit d'une équation chiffrée qui sert d'indicateur objectif et synthétique d'un état de fait 21.

\section{UN NOUVEAU REGARD PUBLIC SUR LA JUSTICE}

Cette reformulation chiffrée de la «crise de la justice»n'est pas propre à cette institution. On le sait, d'autres univers sociaux - particulièrement ceux de la santé et de l'université ( $c f$. classement de Schangaï) - sont touchés de plein fouet par des changements analogues 22 . Ici comme ailleurs, la saillance du chiffre participe de transformations profondes du regard public sur ces institutions traditionnellement marquées par des formes d'auto-régulation à distance de l'État et du marché. Sous l'effet d'un ensemble complexe de dynamiques sociales spécifiques (économiques, journalistiques, administratives, politiques), mais en bien des points convergentes, ces vieux «blocs de granit » se trouvent désormais requis de rendre des comptes. Dans chacun des cas, la mise en chiffre de la justice

17. Il serait erroné pour autant de considérer que l'avènement du chiffre et de la rhétorique de l'objectivité experte qui l'accompagne a fait disparaître ces différents genres classiques de la littérature judiciaire. Les ouvrages des «ténors du barreau ", pénalistes de renom arguant de leur légitimité de prétoire pour intervenir dans les débats judiciaires, les Jean-Marc Varaut, Jean-Denis Bredin, Gilbert Collard, Thierry Levy, Paul Lombard, Daniel Soulez-Larivière ou Jacques Vergès continuent à composer une part substantielle de la production éditoriale en matière judiciaire. Les blogs semblent d'ailleurs donner un nouveau souffle à ce registre d'intervention personnel fondé sur l'expérience directe de l'univers judiciaire : $c f$. http://www.maitre-eolas.fr, http://www.philippebilger.com...

18. Institut Montaigne, Pour la justice, 2004.

19. Union syndicale des magistrats, Le livre blanc sur l'état de la justice 2003, janvier 2004.

20. $C f$. les critiques en ce sens d'Évelyne Serverin, «De la statistique judiciaire civile...», op. cit.

21. Sur ce point, voir Vauchez (Antoine), Willemez (Laurent), La justice face à ses réformateurs (19802006). Entreprises de modernisation, logiques de résistance, PUF, 2007.

22. Sur le monde de la santé, voir les travaux de Pierru (Frédéric), Genèse et usages d'un problème public : la «crise du système de santé » français (1980-2004), Thèse pour le doctorat de science politique, Université de Picardie Jules Verne, 2005. 
fait figure de vecteur privilégié de la montée en puissance d'étalons d'évaluation extrajudiciaires de la qualité de cette institution. Parmi ces groupes sociaux qui participent à cette transformation du regard public sur la justice, il faut assurément évoquer les journalistes. La redéfinition du métier journalistique qui s'opère à partir des années 1980 situe en effet une part de l'excellence professionnelle dans la capacité à pénétrer - au nom d'un « droit de savoir » dont ils se font les porte-parole - des mondes sociaux traditionnellement protégés et marqués par le secret. Parce qu'elle permet de percer l'opacité de ces milieux en s'érigeant au nom du " public » (« la justice est l'affaire de tous ») en arbitre des «bons » et des «mauvais » élèves de la démocratie française, la mobilisation du chiffre des sondages et des palmarès conforte la fonction civique (transparence, devoir d'information) dont nombre de journalistes se sentent aujourd'hui porteurs voire comptables ${ }^{23}$. Préparés par le succès des palmarès hospitaliers, les palmarès judiciaires mais aussi les enquêtes d'opinion arment désormais la critique journalistique des corporatismes et du manque de transparence des professionnels de la justice. Arguant du fait que « la justice est l'affaire de tous » 24, ils dressent un « inquiétant état des lieux » pointant invariablement la « loterie des assises », la «justice inégale », la lenteur des procédures et la défiance de l'opinion à son égard 25.

Mais cette intrusion multiforme des formes d'objectivation chiffrée de l'activité judiciaire ne serait sans doute pas possible sans un ensemble d'outils conceptuels et méthodologiques permettant de penser la machine judiciaire comme une unité économique «comme les autres », autrement dit sans le développement d'une science économique du droit. Longtemps resté cantonné au monde anglo-américain, le mouvement Law and Economics a récemment pris pied sur le territoire français ${ }^{26}$. Le succès de cette approche auprès d'organisations internationales (et d'organisations non gouvernementales) désormais convaincues de l'enjeu économique propre à l'environnement juridique tout comme le poids croissant des logiques économiques sur le «marché du droit » 27 ne sont pas étrangers à cette faveur ${ }^{28}$. Ici aussi, le chiffre est l'instrument essentiel d'un coup de force symbolique qui conduit à repenser l'ensemble des principes (indépendance, inamovibilité...) et traditions juridiques sous l'angle de leur efficience économique. Mais il va de soi cependant que ce sont d'abord les politiques de modernisation de l'État engagées à partir de la fin des années 1980 qui ont contribué de manière décisive à cette fétichisation du chiffre. Parce qu'il est porteur d'une plus grande calculabilité et prévisibilité du fonctionnement de la justice, parce qu'il organise son «gouvernement » autour d'un véritable «tableau de bord » qui en explicite et en systématise les paramètres, le chiffre est un instrument classique de la rationalisation bureaucratique à l'œuvre depuis le début des années 1990. Longtemps considérée comme une administration à part au sein de l'État, justiciable du fait de sa mission particulière de politiques spécifiques,

23. On en trouve un exemple frappant dans la manière dont le chiffre est mobilisé pour transformer les termes et les acteurs des débats sur la justice dans la controverse sur l'ouverture de la commission parlementaire Outreau au nom des premiers taux d'audience télévisée et sondages qui fondaient la revendication des journalistes d'une publicité des débats. $C f$. Vauchez (Antoine), «L'homme, le juge et la 'cage d'acier'. La rationalisation de l'activité judiciaire à l'épreuve du 'moment Outreau' ", in Michel (Hélène) et Willemez (Laurent) dir., $L a$ justice au risque des profanes, CURAPP-PUF, 2007.

24. Thréard (Yves), « La justice, l'affaire de tous », Le Figaro, 18 février 2006, p. 2.

25. Voir, de manière caractéristique, le sondage du CSA du 26 janvier 2006 pour Le Parisien, c'est-à-dire en pleine affaire Outreau, qui fait dire à ce journal que «près des deux tiers des français avouent (sic) qu'ils auraient peur s'ils devaient avoir affaire à la justice. »

26. D'une manière générale sur ce mouvement, voir Kirat (Thierry), L'économie du droit, Repères, La découverte, 1999.

27. Dezalay (Yves), Marchands de droit, Fayard, 1992.

28. Voir le premier numéro spécial de revue juridique consacré à «l'économie de la justice », dans la Revue internationale de droit économique, vol. 13, n² 2, 1999. 
l'institution judiciaire n'échappe pas à la vogue modernisatrice des vingt dernières années. L'investissement financier croissant dans le domaine judiciaire (notamment via la politique de l'aide juridictionnelle) et, d'une manière générale, la place croissante qu'occupent, dans l'activité des gouvernements, les considérations sur l'organisation, les moyens et les méthodes (réunis autour de la thématique de la «réforme de l'État » ${ }^{29}$ ) auront suffit à avoir raison du «splendide isolement » judiciaire. L'application de la loi organique relative aux lois de finances qui soumet la "mission justice » à la même politique d'indicateurs que les 33 autres missions de l'État ne constitue en fait que l'ultime avatar de deux décennies de projets de rationalisation administrative auquel chaque majorité aura apporté sa pierre. Dès lors que l'évaluation de la qualité de la justice devient ainsi avant tout une affaire de quantité - qu'il s'agisse de « rendement » (taux de réponse pénale à assurer), de « célérité » (rapidité de la réponse judiciaire apportée), ou de « coûts » (frais de justice à restreindre) - le chiffre devient le point d'appui indispensable des nouvelles politiques publiques de la justice. Le développement à la Chancellerie et à Bercy d'un ensemble d'outils de gestion permettant un pilotage à distance de la « machine judiciaire » s'accompagne de la réaffirmation de la position des élites et institutions administratives sur les élites et institutions judiciaires (acteurs judiciaires «de terrain »).

Ainsi donc, pour divers qu'ils soient, les usages du chiffre judiciaire sont très variables mais ils forment bien chaque fois l'opérateur voire la clé de voûte des transformations contemporaines du regard public sur la justice.

Ensemble, ils font valoir, sous des formes et à des fins différentes, la possibilité de mesurer et d'objectiver les pratiques judiciaires. En ce sens, ils participent d'un seul et même mouvement de remise en cause de l'auto-régulation sociale de la justice par lequel les élites judiciaires traditionnelles - magistrats en juridiction, avocats, greffiers... - sont sommées de rendre des comptes renonçant par le fait à la singularité et à l'incommensurabilité de ce qu'ils accomplissent.

\section{POLITIQUES DU CHIFFRE}

L'erreur serait sans doute de considérer que le développement de ces nouvelles bases engage une cumulativité permettant à terme de produire un tableau véritablement objectif de «l'état de justice ». Les travaux de sociologie de la statistique invitent en effet à faire le deuil de l'idée qu'il y a aurait une réalité sociale donnée, pré-existante et déjà-là, qu'on pourrait reconstituer à partir du croisement des données. Parce que le chiffre n'existe pas indépendamment de celui qui le produit et lui donne sens, les controverses qui se cristallisent autour de lui ne peuvent s'analyser simplement comme une seule et même recherche $\mathrm{du}$ « bon chiffre », mais forment bien un nouveau terrain d'affrontement où semble se jouer le sort des politiques publiques de justice. Dès lors, en faisant apparaître la double face indissociablement technique et politique du chiffre qui tout à la fois décrit (par la connaissance qu'il permet de construire) et prescrit (par la vision du monde qu'il contribue à faire exister), on cherche ici à rendre compte des transformations du « gouvernement» de la justice.

Toute base de données est en effet porteuse de visions différentes des acteurs, des espaces et des enjeux de la justice. En ce sens, toute construction chiffrée est un coup de force symbolique ou, pour le dire autrement, toute représentation statistique met en jeu la représentation politique du groupe ainsi agrégé, pour quatre raisons au moins. Premièrement, elle fait exister l'« espace de comparabilité » dans lequel se situe l'institution,

29. On renvoie ici aux travaux de Bezès (Philippe), Gouverner l'administration (1962-1997), Thèse de doctorat, Sciences Po Paris, 2002. 
c'est-à-dire ses étalons et ses points de repère ${ }^{30}$, qu'ils soient inter-nationaux dans le cas des classements des organisations internationales, inter-juridictions dans le cas des palmarès journalistiques ou des statistiques judiciaires, inter-magistrats dans le cas de certains des indicateurs de la loi organique relative aux lois de finances (voir, par exemple, l'indicateur « Nombre d'affaires traitées par magistrat ») ou encore inter-institutions pour ce qui est des indices de confiance produits par les instituts de sondage. Deuxièmement, elle conduit à la constitution de moyennes et d'écarts-types. Les mises en série indiquent de ce fait l'espace pertinent d'ajustement (et, partant, de négociation). Troisièmement, du fait des catégories d'analyse qu'elle institue, toute base de données introduit des continuités là où il y a souvent beaucoup d'hétérogénéité et des discontinuités là où on peut trouver des continuum. Ainsi, quand la Chancellerie n'intègre pas les juridictions administratives dans la comptabilisation du budget de la justice et conteste dès lors le classement de la France par la Commission européenne pour l'efficacité de la justice en la matière, elle défend plus qu'un chiffre, mais aussi la spécificité française de la dualité des ordres juridictionnelles, spécificité d'ailleurs maintenue in extremis dans la loi organique relative aux lois de finances elle-même ${ }^{31}$. Quatrièmement, avec le niveau de collecte et le niveau d'agrégation des données (organisme privé, professionnel, syndical, étatique, international...), les bases de données contribuent à établir le niveau pertinent pour la représentation politique du groupe et/ou du problème. De ce point de vue, il y a un écart important entre le classement produit par la Commission européenne pour l'efficacité de la justice qui s'appuie sur des données collectées par les administrations nationales et négocie avec les différents États membres sa nomenclature et le palmarès de la Banque mondiale qui entend court-circuiter les autorités nationales par la constitution dans chaque pays de réseaux d'informateurs « indépendants ».

Dès lors que les conventions statistiques mettent en jeu une représentation des enjeux et des acteurs de la justice, on ne s'étonnera pas qu'une partie des controverses se joue désormais autour voire sur le chiffre. Peut-on "comparer des pommes et des oranges » (critique des « effets de structure») ? Peut-on penser les choses ceteris paribus (critique des «effets réverbère ») ? Quelle est la pertinence des nomenclatures ? Quelle est la représentativité statistique de la base ? Autrement dit, qui a le «bon » ou le «meilleur » chiffre ? Longtemps cantonnée aux cercles spécialisés, la critique interne des données a largement débordé l'espace proprement universitaire. La sincérité des conditions de collecte, le réalisme des choix de nomenclatures, la robustesse des conventions statistiques, les biais de représentativité deviennent souvent aujourd'hui le terrain même des controverses. Autrement dit, une part des débats passe désormais par la mise à l'épreuve du bien-fondé des données elles-mêmes. C'est le cas des critiques de la loi organique relative aux lois de finances qui insistent sur tous les biais qu'introduisent les indicateurs de performance choisis, soit pour leur pauvreté (données sommaires), soit du fait de la disparité géographique du contentieux : "comment comparer un tribunal qui juge proportionnellement plus d'escroqueries avec un tribunal » qui «fait » beaucoup de comparution immédiate s'interroge ainsi Bruno d'Aubusson ${ }^{32}$. La mobilisation qui, en

30. Desrosières (Alain), La politique des grands nombres. Histoire de la raison statistique..., op. cit.

31. 14 octobre 2005, Congrès de l'USM, discours de Pascal Clément, garde des Sceaux : « On peut dire que le budget de la Justice bénéficie d'une place honorable en Europe. La grille d'évaluation du CEPEJ avait laissé à l'appréciation des pays l'expression budget annuel alloué à l'ensemble des tribunaux. Ainsi la France n'avait pas comptabilisé la Justice administrative, alors qu'elle était le plus souvent intégrée chez ses voisins. Ce correctif effectué, le budget de la Justice s'élève à 38,3 euros par habitant au lieu de 28,3, soit un niveau proche de la Norvège et des Pays-Bas, c'est-à-dire une place dans le premier quart du classement. », http://www.presse.justice. gouv.fr/index.php?rubrique=10093\&ssrubrique=10240\&article=11314 (consulté le 15 novembre 2007).

32. Aubusson (Bruno d'), Jean (Jean-Paul), « La LOLF et la mission Justice », Pénombre. Lettre grise, 2006, pp. $43-47$, p. 45. 
France, fait suite au premier rapport Doing business (2004) est également caractéristique de ce point de vue. Tout en se donnant un objectif explicitement politique - « démontrer l'efficacité de certains instruments juridiques inspirés du droit, et notamment de la tradition juridique française »- le consortium constitué à travers le groupe sur l'Attractivité économique du droit (AED) a d'abord fait porter sa critique sur la fragilité des prémisses méthodologiques du classement ${ }^{33}$. En dressant l'inventaire des biais et autres défauts de représentativité du classement, ils travaillent ainsi à établir le déficit de réalité du travail de la Banque mondiale ${ }^{34}$. On s'en doute, ces conflits autour du chiffre et de sa validité relèvent au moins autant d'un point d'honneur technicien (défendre ou critiquer la qualité technique d'une base statistique, d'un sondage ou d'un classement) qu'ils ne révèlent des conflits politiques. Faire la preuve des défauts de forme statistique, c'est aussi priver l'institution qui a produit les chiffres de la capacité de parler au nom de la totalité qu'ils étaient censés représenter ${ }^{35}$. Pour le dire autrement, la mise à l'épreuve de la légitimité d'une institution à prendre part au débat sur la justice passe par celle des données dont elle s'autorise pour «dire » le groupe, son état et ses problèmes. En ce sens, le raffinement statistique croissant des données produites s'analyse aussi comme le fruit de ces luttes sur le chiffre où se joue la capacité d'une base (et de ses producteurs) à représenter une "population" donnée (d'individus, de juridictions, de systèmes juridiques...). Pour faire face aux critiques de ce qui fait figure de prémisse ( «'explosion du contentieux ») de ses politiques de modernisation, la Chancellerie revendique ainsi « un gros investissement sur l'appareil statistique depuis plusieurs années pour isoler dans les comparaisons des délais de jugement au civil ce qui est imputable au volume des effectifs, mais aussi à l'organisation de la juridiction » ${ }^{36}$. De manière similaire, la Banque mondiale a considérablement amendé et enrichi son appareil de mesure de l'attractivité économique au lendemain de son premier rapport lourdement critiqué en France. Autrement dit, derrière ou, plus exactement, dans l'apparente technicité de la discussion chiffrée sur la justice se joue une question politique, celle des acteurs et des catégories de la réforme, - et donc du gouvernement -, de la justice. Mieux, on peut faire l'hypothèse que c'est la technicité même de ces débats qui permet d'engager des discussions sur des points politiquement aussi sensibles que celui du droit de regard d'organisations internationales sur le fonctionnement d'un ordre juridique national réputé souverain ou celui du droit de regard de la Chancellerie (et de l'administration centrale au sens large) sur les pratiques judiciaires réputées indépendantes. Dès lors que s'y joue des questions politiques aussi critiques, on ne s'étonnera pas que la technique quantitative devienne un des langages privilégiés des débats publics sur la justice. Il ne faudrait pas pour autant considérer que ne se joue ici qu'une simple reformulation en termes chiffrés de conflits et de clivages pré-existants. En accompagnant l'intrusion d'experts et de types d'expertise qui lui étaient jusque là largement (ou complètement) étrangers, qu'ils soient statisticiens, instituts de sondage, hauts fonctionnaires du ministère des finances, gestionnaires de la Chancellerie, experts en finances publiques, comptables, économistes et consultants, le chiffre contribue à transformer en profondeur le périmètre du débat. Et c'est là sans doute l'un des effets les plus inaperçus de cette technicisation que de s'accompagner d'une recomposition des savoirs et des pouvoirs au sein de l'institution judiciaire elle-même.

33. Marais (Bertrand du) dir., Des indicateurs pour mesurer le droit? Les limites méthodologiques des rapports Doing business, La documentation française, 2006.

34. «Le programme de recherche sur l'attractivité économique du droit », La lettre de l'AED, n4, 2007, p. 4.

35. Sur ces liens entre représentation statistique et représentation politique, voir Thévenot (Laurent), «Statistique et politique : La normalité du collectif », Politix, n²5, 1994, pp. 5-20.

36. On pourrait dire de même à propos du «taux de réponse pénale », nouvel indicateur censé offrir une vue de la «réalité » de la réponse de l'État plus fine que le «taux de classement sans suite » indicateur plus grossier (et partant plus fragile) qui agrégeait l'activité des services de police et l'activité propre des parquets. 